Methods Between July and December 2018, a cross-sectional study among long-distance truck drivers was carried out in four union halls in Mysore, Karnataka. After undergoing an informed consent process, all participants answered an interviewer-administered questionnaire. Blood samples were screened for HIV, Hepatitis B and HCV (ERBA ELISA, Trans Asia Biomedicals Ltd, Mumbai, India). Detection of Treponemal Antibodies was carried out using a Rapid Plasma Reagen test kit (ARKRAY Healthcare Pvt. Ltd., India).

Results The prevalence of HIV infection was 2.2\% (95\% CI: 1.1-4.0); Hepatitis B, 2.6\% (95\%CI: 1.4-4.6); and syphilis, 1\% (95\%CI: $0.3-2.5$ ). There were no cases of HCV detected. Prevalence of any of the STIs was 5.9\% (95\%CI: 3.9-8.5). The mean age was 43.9 years $(\mathrm{SD} \pm 7.49)$, 93\% were married, $87 \%$ had no education or less than 10 years of education. About $81 \%$ reported their religion as Hindus. A majority (61.5\%) belonged to a backward caste, scheduled caste or scheduled tribe. Religion and income were significantly associated with being diagnosed with any STIs.

Conclusion While prevalence for HIV and common STI appear to be going down in this population, rates are still moderately higher than those found in the general population. There is a need for continued interventions to prevent truckers from bridging HIV and Hepatitis to the general population.

Disclosure No significant relationships.

\section{P192 HEALTHCARE WORKER RELATED STIGMA AND DISCRIMINATION TOWARDS PEOPLE LIVING WITH HIV IN ONE OF THE GOVERNMENT HOSPITAL IN BOHOL}

Lydette Galimba*. Gov. Celestino Gallares Memorial Hospital, Family and Community Medicine, Agbilaran City, Philippines

\subsection{6/sextrans-2019-sti.344}

Background Stigma and discrimination of people living with HIV (PLHIV) are one of the most under researched area of HIV and provided a large gap in the HIV evidence bases. The major complication of this stigma becomes evident in the health seeking behavior of patients suspected to have HIV.The study primarily aimed at assessing the stigma and discrimination to the people living with Human Immunodeficiency Virus among health care workers at Governor Celestino Gallares Memorial Hospital.

Methods This study utilized a descriptive design. It made use of a standardized questionnaire assessing the personal profile of the respondents in terms of service affiliation and trainings on PLHIV stigma and discrimination received, and the seven identified domains of stigma and discrimination.

Results The findings revealed high levels of stigma and discrimination among the respondents on fear related to drawing of blood from a patient living with HIV or AIDS patient $81.59 \%$, observation on healthcare workers providing poorer quality of care to a patient living with or thought to be living with HIV than other patients $77.04 \%$ and dressing the wounds of a patient living with HIV or AIDS patient $75.23 \%$. On the other hand, low percentage was registered on attitude towards key populations ranging from 5.5\%-13.61\%. Further, the study have revealed that the institution have only $8.2 \%$ of personnel who were trained on PLHIV stigma and discrimination.
Conclusion Grounding on the findings, the researcher highly recommends integration of Stigma and Discrimination Orientation/Trainings on hospital personnel catering to key populations and PLHIV to reduce the current level of stigma and discrimination. Increasing the understanding and correcting misconceptions on PLHIV and its accompanying stigma and discrimination to health care personnel through orientation and trainings would provide better avenue on the provision of high quality HIV access to the targeted populations.

Disclosure No significant relationships.

\section{P193 ASSESSING WOMEN PERSPECTIVES ON HIV TRANSMISSION RISK IN RURAL UPPER WEST REGION OF GHANA: A QUALITATIVE STUDY}

${ }^{1}$ Alexander Laar*, ${ }^{2}$ Syvester Isang, ${ }^{3}$ Benjamin Baguune, ${ }^{3}$ Emmanuel Bekyieriya. ${ }^{1}$ University of Newcastle, Australia, Public Health and Medicine, Newcastle, Australia; ${ }^{2}$ Ghana School of Law, Kwame Nkrumah University of Science Technology, Kumasi, Ghana., Law, Kumasi, Ghana; ${ }^{3}$ School of Hygiene, Environmental Health Programme, Ministry of Health, Tamale, Ghana, Health, Tamale, Ghana

\subsection{6/sextrans-2019-sti.345}

Background Despite the steps taken by the Government of Ghana towards preventing or eliminating mother-to-child transmission (MTCT) of HIV in line with key international recommendations and guidelines, MTCT of HIV prevalence rate still remain high in the rural Upper West region of Ghana. This study explored the perspectives of rural women on circumstances that may facilitate HIV transmission and prevention in a high MTCT of HIV prevalence districts.

Methods Qualitative methods involving 6 focus group discussions were conducted using semi-structured interview guide in three rural districts. A purposeful sampling method was used to select eligible participants. The data were analyzed using a thematic framework approach.

Results The study findings show that all participants have heard of HIV and AIDS. Both pregnant women and breastfeeding mothers' had knowledge that HIV can be transmitted from the mother to the child. With regards to specific transmission routes and stages of transmission, during pregnancy, during labour and delivery and through breastfeeding were mentioned. However, knowledge on MTCT was inadequate since some participants did know that wet-nursing was one of the transmission routes. Wet-nursing practices were prevalent in these communities. Some reasons given for the practice included initial breast milk of the mother not good for the baby's consumption or health because it is dirty or contaminated. To get rid of the initial milk, a breastfeeding mother is engaged to breastfeed (wet-nurse) the new-born as custom demands until the so-called dirty breast milk is gotten rid of. Other traditional risky practices were circumcision and giving of tribal marks to the newborn by traditional birth attendants.

Conclusion The findings of our study revealed that traditional practices which could have negative outcomes on maternal and child health are still carried out in the study settings. Interventions that seek to change social behaviours by addressing cultural norms is desirable.

Disclosure No significant relationships. 